\title{
Patterns of Brain Structural Changes in First-Contact, Antipsychotic Drug-Naïve Patients with Schizophrenia
}

\author{
M. Filippi, E. Canu, R. Gasparotti, F. Agosta, P. Valsecchi, G. Lodoli, A. Galluzzo, G. Comi, and E. Sacchetti
}

\begin{abstract}
BACKGROUND AND PURPOSE: Previous studies have suggested that structural changes do occur in the brain of patients with schizophrenia compared with healthy control participants. However, findings from such studies are inconclusive, probably because of the different methodologic approaches, the clinical heterogeneity of patient samples, and also the fact that patients enrolled were treated with antipsychotic drugs. The aim of this study was to investigate brain GM volumes and intrinsic structural WM changes in first-contact, antipsychotic drug-naïve patients with schizophrenia.
\end{abstract}

MATERIALS AND METHODS: A total of 43 first-contact, drug-naïve, patients with schizophrenia and 17 age-matched control participants were studied. All participants underwent T1-weighted MR imaging and DTI scans. Voxel-based morphometry and tract-based spatial statistics were used to compare GM volumes and WM DTI metrics between groups. MR imaging measures were correlated with the duration of the untreated psychosis and the clinical positive and negative symptoms.

RESULTS: Compared with control participants, patients with schizophrenia showed smaller volumes of the temporal, parietal, and occipital GM, and a pattern of decreased mean diffusivity and increased fractional anisotropy in the brain stem and cerebellum bilaterally, interhemispheric and cortico-cortical connections bilaterally, and right anterior and posterior limb of the internal capsule. In patients, decreased mean diffusivity and increased fractional anisotropy in several brain regions were related to a longer duration of the untreated psychosis and the severity of positive symptoms.

CONCLUSIONS: First-contact, drug-naïve, patients with schizophrenia present with volumetric and DTI changes, which correlated with their clinical features. This study increases our knowledge on the neural networks involved in the pathophysiologic mechanisms of schizophrenia.

ABBREVIATIONS: DARTEL = Diffeomorphic Anatomical Registration through Exponentiated Lie Algebra; DUP $=$ duration of the untreated psychosis; FA = fractional anisotropy; MD = mean diffusivity; PANSS = Positive and Negative Syndrome Scales; MNI = Montreal Neurological Institute; TBSS = tract-based spatial statistics

l recent years, interest in the physiopathologic mechanisms of schizophrenia has increased dramatically. As normal brain functions are served by networks of cortical and subcortical areas,

Received November 16, 2012; accepted after revision February 4, 2013.

From the Neuroimaging Research Unit (M.F., E.C., F.A.) Institute of Experimental Neurology, Division of Neuroscience, and Department of Neurology (M.F., G.C.) San Raffaele Scientific Institute, Vita-Salute San Raffaele University, Milan, Italy; Department of Diagnostic Imaging (R.G., G.L.), Neuroradiology Unit, Brescia University School of Medicine, Spedali Civili, Brescia, Italy; Department of Mental Health (P.V., A.G., E.S.), Spedali Civili, Brescia, Italy; University Psychiatric Unit (P.V., E.S.), Brescia University School of Medicine, Brescia, Italy; Centre on Behavioural and Neurodegenerative Disorders (E.S.), Brescia University and EULO, Brescia, Italy.

Please address correspondence to Prof. Massimo Filippi, Neuroimaging Research Unit, Institute of Experimental Neurology, Division of Neuroscience, San Raffaele Scientific Institute, Vita-Salute San Raffaele University, Via Olgettina, 60, $20132 \mathrm{Mi}$ lan, Italy; e-mail: m.filippi@hsr.it

三 Indicates article with an on-line appendix

Indicates article with supplemental on-line figure

http://dx.doi.org/10.3174/ajnr.A3583 disturbed communication ("dysconnectivity") within and between brain regions may be the core pathologic feature of schizophrenia. ${ }^{1}$ In this context, the contribution of neuroimaging techniques has proved to be relevant. Structural MR imaging studies have shown that reductions of GM volume occur in the brain of patients with schizophrenia compared with healthy control participants, though increased GM volumes of some subcortical regions have also been reported. ${ }^{2}$ DTI studies of schizophrenic patients have disclosed an abnormal organization and integrity of several WM tracts of the brain. However, results are conflicting regarding which tracts are affected, with some DTI studies showing a marked involvement of the anterior pattern of brain WM regions in patients with schizophrenia, and others describing a posterior pattern of WM changes or even no difference compared with control participants. ${ }^{3}$

The inconclusive findings of previous studies are probably the result of the clinical heterogeneity of the samples and/or the dif- 
Table 1: Sociodemographic and clinical features of patients and control participants

\begin{tabular}{lccc}
\hline & \multicolumn{1}{c}{ Patients } & Control Participants & \multicolumn{1}{c}{$P^{\mathbf{a}}$} \\
\hline No. & 43 & 17 & \\
Age (range) & $29.3 \pm 7.4(17-44)$ & $30.7 \pm 8.6(19-50)$ & .687 \\
Women (\%) & $19(44 \%)$ & $11(65 \%)$ & .252 \\
Education (y) & $11.0 \pm 3.4(1-18)$ & $15.2 \pm 3.2(9-19)$ & $<.001$ \\
DUP (months) & $7.9 \pm 9.7(1-40)$ & - & - \\
PANSS total score & $100.8 \pm 14.7(64-125)$ & - & - \\
PANSS positive score & $28.2 \pm 6.4(15-49)$ & - & - \\
PANSS negative score & $23.0 \pm 5.6(12-34)$ & - & - \\
PANSS composite score & $5.2 \pm 7.4(-8-21)$ & - & - \\
\hline
\end{tabular}

${ }^{a}$ Mann-Whitney and Fisher exact test. Composite score (PANSS positive-PANSS negative score). the enrollment; no family ties with other enrolled participants; a negative history of seizures and head trauma with loss of consciousness; no concomitant major medical conditions; Mini-Mental State Examination $^{11}$ score $>24$; an IQ score on the Wechsler Adult Intelligence Scale-Revised $^{12}>75$; and no other causes of focal or diffuse brain damage, including lacunae, and extensive cerebrovascular disorders at routine MR imaging.

Furthermore, patients were included if they had a current $D i$ agnostic and Statistical Manual of Mental Disorders, (DSM-IV), diagnosis of schizophrenia ${ }^{13}$; no lifetime comorbidities with DSM$I V$, Axis I disorders; a first-in-lifetime psychiatric contact; a level of understanding judged sufficient to give informed consent; and willingness to undergo an MR imaging examination before starting an antipsychotic drug. The diagnosis of schizophrenia and the exclusion of Axis I comorbidities were based on a detailed clinical interview complemented by a revision of all medical records.

For control participants, the group-specific inclusion/exclusion criteria were no lifetime evidence of any DSM-IV, Axis I disorder; a negative family history for psychosis and mood disorders in first-degree relatives; and willingness to perform an MR imaging examination.

A total of 43 patients with schizophrenia and 17 control participants were enrolled (Table 1). After complete description of the study to the participants, written informed consent was obtained. Participants also received an explicit guarantee of anonymity. ${ }^{14}$

\section{Clinical Assessment}

Participants were evaluated by a team of qualified and experienced psychiatrists, blinded to MR imaging data. Direct information from the patients, together with systematic interviews of at least 1 family member, was collected to date the age of onset of schizophrenia, operationally identified as when the first psychotic symptom with deterioration of function emerged. The interval elapsing between the first symptom and the first psychiatric contact defined the DUP. ${ }^{15}$ The Positive and Negative Syndrome Scales (PANSS) ${ }^{16}$ were administered to the patients.

\section{MR Imaging Study}

Within 2 weeks from the first clinical visit, brain MR imaging scans were obtained by use of $1.5 \mathrm{~T}$ scanner. A detailed description of the MR imaging study is provided in the On-line Appendix. Tract-based spatial statistics (TBSS) was used to perform the multisubject DTI analysis. Voxel-based morphometry was run to assess GM volumes, by use of Statistical Parametric Mapping 8 (Wellcome Department of Imaging Neuroscience, London, UK).

\section{Statistical Analysis}

Demographic and clinical variables were compared between groups by use of the Fisher exact test, the Mann-Whitney $U$ test, or the Kruskal-Wallis test as appropriate $(P<.05$; SPSS 13.0; IBM, Armonk, New York). 

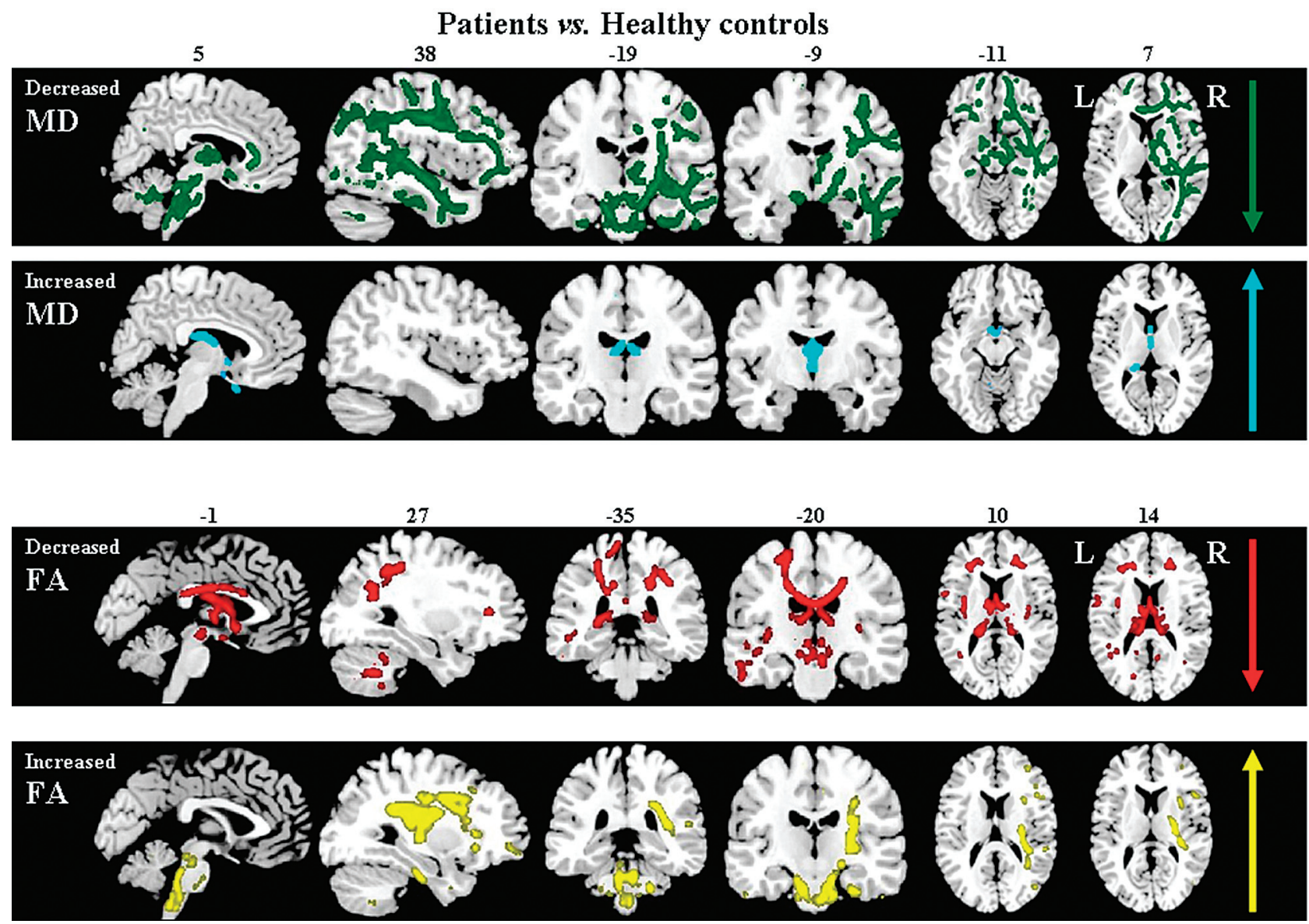

FIG 1. TBSS results in patients compared with healthy control participants. Voxelwise group differences are shown in cyan (increased MD), green (decreased MD), red (decreased FA), and yellow (increased FA). Results are overlaid on the sagittal, coronal, and axial sections of the Montreal Neurological Institute standard brain in neurologic convention (right is right) and are displayed at permutation-based $P<.05$, uncorrected.

DTI voxelwise statistics were performed by use of a permutation-based inference tool for nonparametric statistical thresholding. ${ }^{17} \mathrm{MD}, \mathrm{FA}$, axial, and radial diffusivity values within the skeleton were tested between groups by use of a permutation-based 2-sample $t$ test adjusted for age. ${ }^{17}$ Statistical maps were thresholded at $P<.05$ uncorrected for multiple comparisons.

Analyses of covariance were performed to assess GM volume differences between groups, with adjustment for age and total intracranial volume. Results were tested at $P<.001$ uncorrected, within at least 50 contiguous voxels. Furthermore, maps of the average percentage GM tissue loss in patients vs control participants were computed on the basis of the ratio, at each GM point, between the mean GM attenuation value at that point in the patient group and the corresponding mean GM attenuation value of the control group. The mean percentage GM tissue loss of clusters showing a significant between-group difference was also measured.

In patients, the associations of DUP and PANSS scores with DTI variables and GM volumes were tested by regression models in the FMRIB Software Library (http://www.fmrib.ox.ac.uk/fsl) and Statistical Parametric Mapping 8, respectively. Results were assessed at $P<.05$ for WM and $P<.001$ within 50 voxels for GM, uncorrected. The mean FA and MD values of the skeletonized voxels showing a significant correlation with clinical features were

Filippi Jan 2014 www.ajnr.org

calculated. Then, mean FA and MD values were correlated with DUP and PANSS by use of the Pearson coefficient adjusted for the participant age $(P<.05$; SPSS).

\section{RESULTS}

\section{Demographic and Clinical Features}

Compared with control participants, patients had similar age and sex but differed in length of education (Table 1).

\section{WM Damage: TBSS}

Patients compared with control participants showed an increased MD in the fornix and thalamic radiations bilaterally, and right olfactory bulb (Fig 1). In patients vs control participants, decreased MD was found in the posterior cerebellar lobe bilaterally, cerebral peduncles, pons and medulla oblongata, middle and inferior cerebellar peduncles, genu of the corpus callosum, middle cingulum, uncinate and parahippocampal tracts bilaterally, and right anterior and posterior limbs of the internal capsule, right thalamic radiations, right inferior fronto-occipital fasciculus, and right superior and inferior longitudinal fasciculi (Fig 1). Compared with control participants, patients had a decreased FA in the cerebral peduncles, thalamic radiations, fornix, corona radiata (with a left-side predominance), corpus callosum, superior longitudinal fasciculus (with a left-side predominance) bilaterally, 
Table 2: Smaller GM volumes in patients vs control participants

\begin{tabular}{|c|c|c|c|c|c|c|}
\hline \multirow{2}{*}{$\begin{array}{l}\text { Cluster Size } \\
\text { (no. of Voxels) }\end{array}$} & \multirow[b]{2}{*}{ Brain Region } & \multicolumn{3}{|c|}{$\begin{array}{l}\text { Stereotaxic Coordinates } \\
\text { (mm) }\end{array}$} & \multirow[b]{2}{*}{ T Value } & \multirow{2}{*}{$\begin{array}{l}\text { Percentage of } \\
\text { Tissue Loss }\end{array}$} \\
\hline & & $\mathbf{x}$ & y & z & & \\
\hline 322 & Right fusiform gyrus & 37 & -54 & -7 & 5.01 & 14 \\
\hline 220 & Left middle temporal lobe & -47 & -54 & 19 & 4.08 & 14 \\
\hline 53 & Left middle temporal lobe & -60 & -8 & -13 & 3.65 & 8 \\
\hline 52 & Left postcentral gyrus & -37 & -40 & 49 & 4.35 & 11 \\
\hline 82 & Right postcentral gyrus & 37 & -33 & 54 & 4.25 & 12 \\
\hline 379 & Left precuneus & -11 & -46 & 42 & 4.07 & 12 \\
\hline 51 & Right middle cingulate & 13 & -54 & 33 & 3.66 & 9 \\
\hline 85 & Left superior occipital gyrus & -18 & -97 & 8 & 3.77 & 17 \\
\hline 154 & Left cerebellum & -15 & -42 & -62 & 3.75 & 12 \\
\hline 120 & Left cerebellum & -34 & -61 & -32 & 3.59 & 6 \\
\hline
\end{tabular}

Note:- No regions of increased GM volume were found in patients vs control participants.

\section{Patients ws. Healthy controls}

$-18$

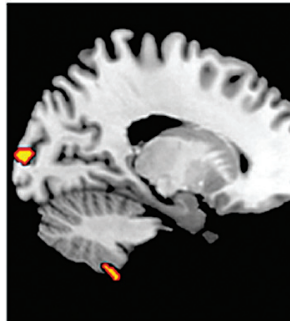

$-2$

$-54$

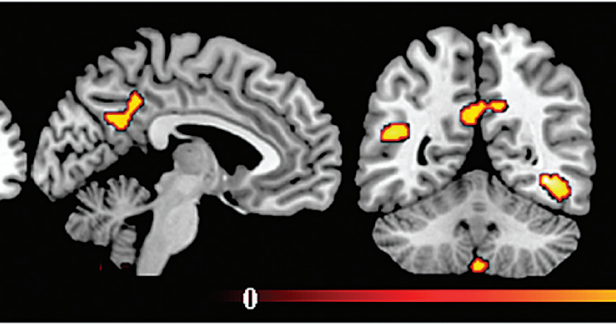

$-37$

$-7$

32

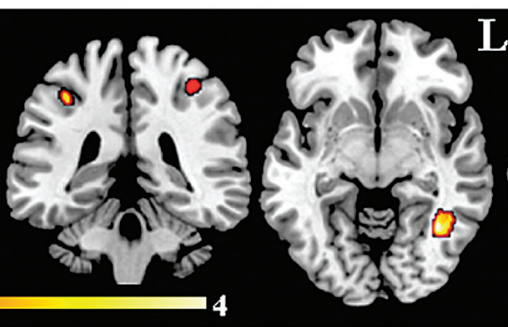

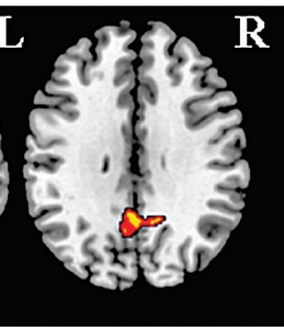

$-37$

$-7$

32

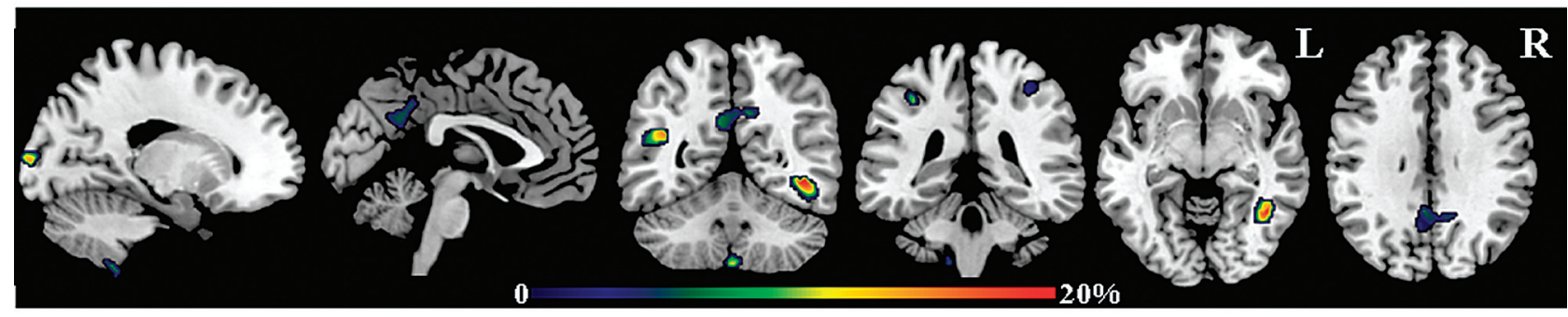

FIG 2. Voxel-based morphometry results showing smaller GM volumes in patients compared with healthy control participants. Results are overlaid on the sagittal, coronal, and axial sections of the MNI standard brain in neurologic convention (right is right) and are displayed at $P<$ .001 , uncorrected within at least 50 contiguous voxels. Color bars denote T values (on the top) and percentages of GM reduction (at the bottom). No region of increased GM volume was found in patients compared with control participants.

right superior cerebellar peduncle and posterior cerebellar lobe, and left olfactory bulb, left posterior parahippocampal tract, and left inferior fronto-occipital fasciculus (Fig 1). Compared with control participants, patients also had regions of increased FA in the cerebral peduncles, pons and medulla oblongata, middle and superior cerebellar peduncles bilaterally, and right posterior limb of the internal capsule, right external capsule, right frontoparietal part of the superior longitudinal fasciculus, and right parahippocampal tract (Fig 1).

Axial and radial diffusivity patterns resembled those of MD and FA in all contrasts. In all comparisons, patterns of decreased axial diffusivity mirrored those of reduced MD, while patterns of increased axial diffusivity reflected those of increased MD and decreased FA (On-line Figure). Patterns of decreased radial diffusivity reflected those of increased FA, whereas increased radial diffusivity reflected those of increased MD (On-line Figure).

\section{GM Volumes: Voxel-Based Morphometry}

Compared with control participants, patients showed smaller GM volumes of the bilateral postcentral gyrus; right fusiform gyrus and middle cingulate cortex; and left middle temporal and superior occipital gyrus, precuneus, and cerebellum (Table 2, Fig 2). In these regions, patients with schizophrenia had up to 20\% GM loss relative to healthy control participants. No regions of increased GM volumes were found in patients compared with control participants.

\section{Relationship between Clinical Features and GM and WM Damage}

DUP. In patients, a negative relationship was found between DUP and MD values of the cerebral peduncles, pons and medulla oblongata, middle cingulum, parahippocampal tracts and inferior longitudinal fasciculi bilaterally, and right posterior limb of 


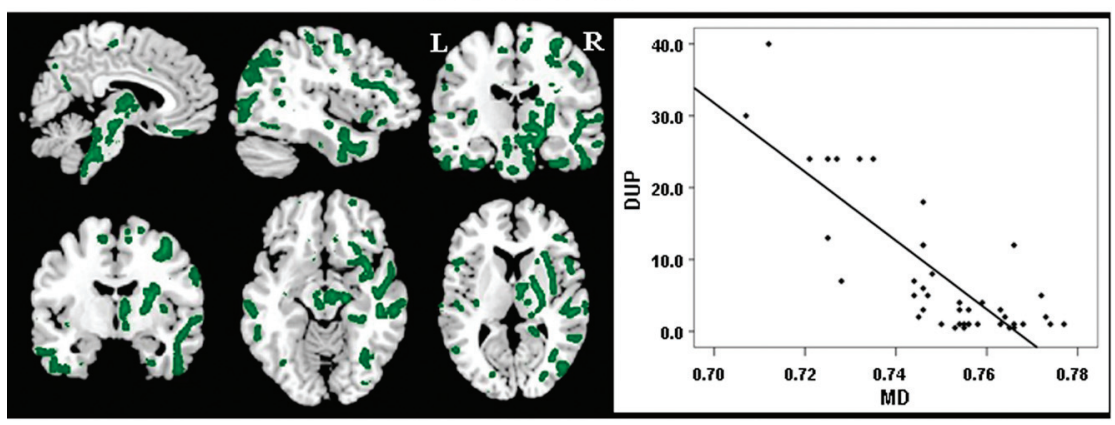

FA vs. DUP

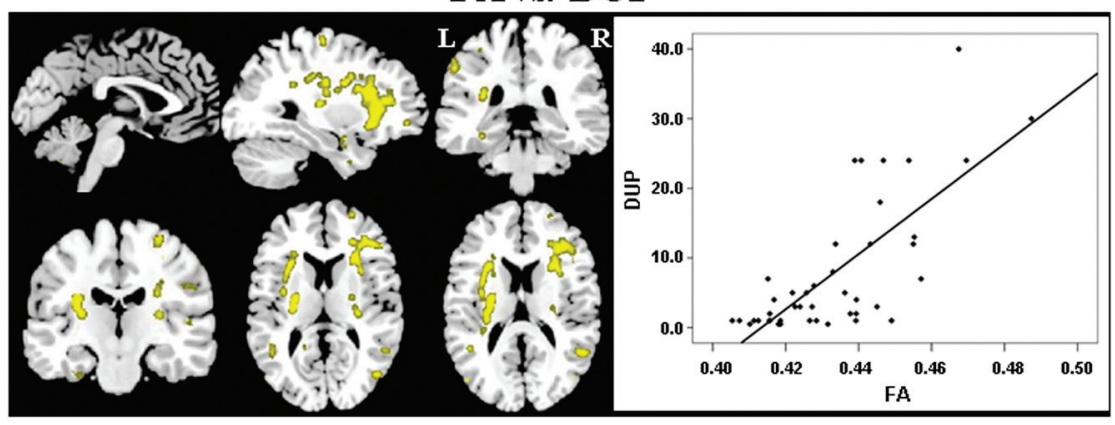

B

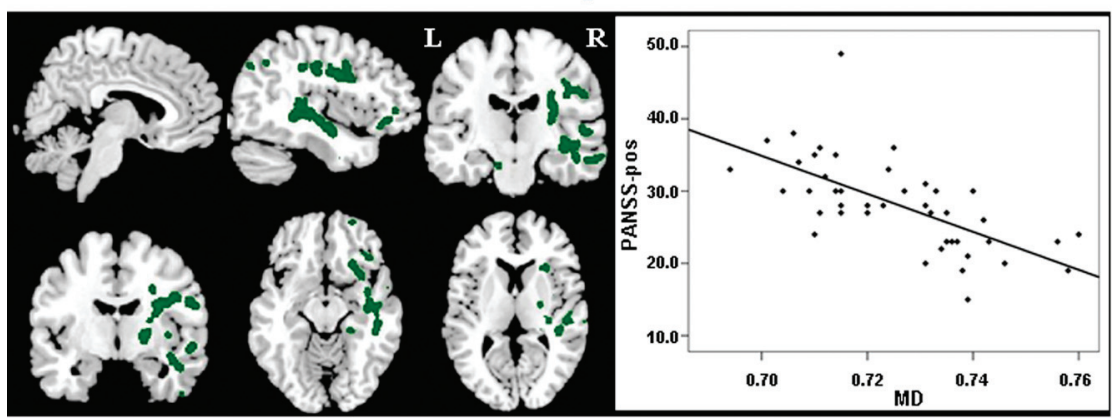

FIG 3. A, Relationships of the DUP with MD (green, negative correlation) and FA (yellow, positive correlation). B, Relationships between the PANSS-positive scores and MD (negative correlation). Results are overlaid on the sagittal, coronal, and axial sections of the MNI standard brain in neurologic convention (right is right) and are displayed at permutation-based $P<.05$, uncorrected.

the internal capsule, right thalamic radiations, right inferior fronto-occipital, and right uncinate fasciculi (Fig $3 A$ ). In patients, a positive relationship was found between DUP and FA values of the posterior limb of the internal capsule, superior longitudinal (with a left-side predominance) and inferior fronto-occipital fasciculi bilaterally, and the right uncinate fasciculi (Fig $3 A$ ). Pearson correlation coefficients were -0.81 for MD values and 0.82 for FA values $(P<.001)$.

PANSS Scores. In patients with schizophrenia, MD values of the right middle and inferior cerebellar peduncles, uncinate and parahippocampal tracts, inferior longitudinal fasciculus, posterior limb of the internal capsule, inferior fronto-occipital and superior longitudinal fasciculi, and the left posterior cerebellar lobe were inversely related with the PANSS positive score (Fig 3B). The Pearson correlation coefficient was $-0.69(P<.001)$.

\section{DISCUSSION}

To our knowledge, this study is the first to investigate both microstructural WM and volumetric GM changes in a large group of first-contact, antipsychotic drug-naïve patients with schizophrenia. We observed that the brain of patients with schizophrenia is characterized by the coexistence of WM microstructural changes and reduced GM volumes in several brain regions since the clinical onset of the disorder. The inclusion of patients at the first psychotic event further highlights the precocity of the observed brain changes. Compared with control participants, patients with schizophrenia showed WM changes, in both higher and lower diffusion, of the following networks: the limbic system, the interhemispheric connections, the cortico-cortical systems, the motor system, and the cerebello-thalamo-cortical circuit. Patients with schizophrenia also had reduced GM volume mainly of the posterior brain regions. MR imaging changes observed in our patients may be the structural correlates of the "hypoconnectivity" and "hyperconnectivity" between brain regions that have been proposed to characterize cortical and subcortical connections in schizophrenia. ${ }^{18}$ The potential role of structural brain "disconnectivity" 18 in determination of the clinical picture of schizophrenia is further suggested by the correlation analysis. Indeed, in patients with schizophrenia, we observed a positive correlation between DUP and FA, and a negative relationship of DUP and positive symptoms with MD values of the motor and the cortico-cortical systems, suggesting the dysfunctional nature of the structural "hyperconnectivity" of these systems in untreated patients.

\section{WM Findings}

Compared with control participants, patients with schizophrenia showed decreased FA and increased MD in several regions corresponding to those observed in other studies that investigated never-medicated schizophrenic patients between 18 and 45 years old. ${ }^{4-8}$ Previous authors have found lower FA values in the fornix, ${ }^{7}$ thalamic radiations, ${ }^{8}$ corpus callosum,,${ }^{4,6,8}$ superior longitudinal $^{7,8}$ and inferior fronto-occipital fasciculi, ${ }^{4,8}$ and the corticospinal tracts ${ }^{8}$ relative to healthy subjects. Our findings are also in agreement with previous studies in medicated schizophrenic patients showing decreased FA values in the corpus callosum, thalamic radiations, and superior longitudinal fasciculus (for a com- 
prehensive review of WM findings in schizophrenia, please see Kyriakopoulos and Frangou et $\mathrm{al}^{19}$ and Peters et $\mathrm{al}^{1}$ ). Postmortem studies have identified WM alterations in schizophrenia, which include microstructural abnormalities of the myelin and oligodendrocytes that may represent the pathologic substrate of the observed MD increases and FA decreases in the MR imaging results of our study and in previous MR imaging reports. ${ }^{20}$

Different from other studies of first-episode antipsychotic drug-naïve patients with schizophrenia, ${ }^{4-9}$ we observed that patients also showed regions of increased FA and decreased MD values, likely reflecting structural "hyperconnectivity," 18 mainly in the brain stem, cerebellum, and in the interhemispheric and cortico-cortical WM connections. Some studies of patients with schizophrenia have found a similar pattern of DTI changes. ${ }^{21,22}$ It is interesting to note that a recent study detected both increased and decreased FA in untreated patients with schizophrenia in the corticopontine-cerebellar circuit, ${ }^{21}$ with the most inferior sections of the corticopontine tracts and the middle cerebellar peduncles showing higher FA values in patients compared with control participants (ie, exactly the same pattern that we observed in our present study; see the sagittal sections of FA: $\times-1$ and MD: $\times 5$, Fig 1). Another DTI study of 12 first-episode patients with schizophrenia found an increased intervoxel coherence, which corresponds to high FA, in the thalamic radiations. ${ }^{23}$ The pathophysiologic correlates of decreased MD and increased FA in patients with schizophrenia can be diverse. First, an increased WM fiber attenuation in patients with schizophrenia vs healthy participants is observed, as shown by postmortem studies investigating the WM architecture of patients with schizophrenia. ${ }^{24}$ Second, there is decreased integrity of the corticopontine inhibitory GABAergic projections that may result in an increased axonal remyelination downstream, ${ }^{21}$ which, in turn, may cause a paradoxic decrease of MD and increase of FA as observed in our study. Third, an alternative, but not mutually exclusive, explanation may be related to the dopamine hypothesis of schizophrenia, ${ }^{25}$ according to which a hyperdopaminergic status could lead to "overdemanding" neuronal activity able to initiate an increase of useless WM connections and, clinically, induce aberrant assignments of motivational salience to objects, people, and actions.

In patients with schizophrenia, we observed that the DUP correlated negatively with MD values and positively with FA values. In addition, we also found a negative correlation between MD of some cerebellar and cerebral regions and the severity of the clinical positive manifestations. A relationship between increased FA values of the fronto-occipital and temporolimbic tracts and more severe positive symptoms has been previously observed in nevermedicated patients with schizophrenia ${ }^{5,10}$ and in medicated patients with schizophrenia with a history of auditory hallucinations and other positive symptoms. ${ }^{26-28}$ Overall, these findings suggest that the observed pattern of structural WM changes in patients with schizophrenia is already present before treatment initiation and is associated with the occurrence of positive clinical manifestations.

It is worth noting that MD and FA changes may be secondary to modifications of diffusion either parallel (axial diffusivity) or perpendicular (radial diffusivity) to the principal direction of the tensor. ${ }^{29}$ Analysis of directional diffusivities may provide addi- tional information on the underlying mechanisms of WM integrity loss. ${ }^{29}$ Myelin breakdown is likely to be associated with increased diffusivity perpendicular to the WM tract, whereas axonal damage has been suggested to reflect diffusivity changes parallel to the primary fiber orientation. ${ }^{30,31}$ However, although study of axial and radial diffusivity helps to interpret MD and FA changes, radiopathologic correlations on the basis of investigation of these metrics remain controversial. ${ }^{32}$

\section{GM Findings}

Drug-naïve patients with schizophrenia had reduced volumes of the temporal, parietal, and occipital cortices and cerebellum. Only a few studies have investigated GM volumes in patients with schizophrenia at the first psychotic episode and who are antipsychotic-naïve, and have shown a pattern of GM volume loss ${ }^{33}$ similar to the one we observed.

Reduced volume of the fusiform gyrus ( $14 \%$ of tissue loss) is in line with the results of a neuropathologic study of patients with schizophrenia showing an $18 \%$ reduction of neuron attenuation in layers III and V of this region. ${ }^{34}$ The fusiform gyrus plays a relevant role in face-processing integrating perception, memory, and emotion. ${ }^{35}$ Reduced GM volume of this region may, therefore, be linked to a failure in facial identification and emotion and, thus, may lead to typical impairment of the empathic social interaction known to occur in these patients. ${ }^{35}$ Previous structural MR imaging studies in medicated patients with schizophrenia also showed smaller volumes of the posterior cerebellum. ${ }^{36}$ Cerebellar damage has been shown to be associated with negative symptoms and mood and behavioral dysregulation. ${ }^{37}$ Reduced volume of the precuneus was also found in patients with schizophrenia and was associated with delusions of control. ${ }^{38}$ We also detected reduced volume of the superior occipital region, which subtends perceptual functions known to be frequently altered in schizophrenia. In addition, compared with control participants, patients with schizophrenia showed smaller volumes of the middle temporal lobe. This region has been found to be atrophic in a study investigating GM attenuation in high-risk patients in whom psychosis later developed. ${ }^{39}$

\section{Study Strengths and Limitations}

Having avoided the potential effects played by the chronicity of the disorder on brain MR imaging results and those associated with concomitant treatment, we believe that the presence of multiple structural brain changes in our patients may be conceivably seen as an early phenotypic expression of the processes involved in the pathophysiologic mechanisms of schizophrenia. However, our study was cross-sectional and, therefore, did not allow us to disentangle the role of neurodevelopmental and neurodegenerative abnormalities, which may both result in the observed MR imaging changes. The patients in our study did not undergo comprehensive cognitive testing and were not educationally matched with healthy control participants. However, the inclusion of participants with a Mini-Mental State Examination score $>24$ and IQ $>75$ should have reduced the effect of intellectual functions and educational level on the MR imaging results. In addition, although the exclusion of patients with a lifetime history of Axis I disorders precludes a major effect of cannabis abuse on our find- 
ings, a possible influence of a sporadic use of cannabis during adolescence cannot be excluded. ${ }^{1}$ In patients with recent-onset schizophrenia, a study found that cannabis use before age 17 years was related with increased FA in several brain regions, including the uncinate fasciculus, internal capsule, and frontal WM, compared with patients without a history cannabis use and compared with control participants. ${ }^{40}$ In another study, the authors found that compared with cannabis-naïve patients with schizophrenia, patients who used cannabis showed increased FA in the splenium of the corpus callosum. ${ }^{41}$ Evidence of an association between increased FA and cannabis use comes from studies of cannabis users without major psychiatric illnesses, showing increased $\mathrm{FA}^{42,43}$ and reduced $\mathrm{MD}^{42}$ of several WM regions in these participants. All of these findings are compatible with the ability of the cannabinoid receptor stimulation to increase the oligodendrocyte transcription factor Olig2 as well as the expression of myelin basic protein. ${ }^{44}$

Some technical limitations of this study also need to be considered. The diffusion tensor model is currently the framework most commonly used to relate the diffusion signal to the direction of the fibers. However, this model has been shown to be inadequate in voxels containing multiple fiber orientations (eg, "crossing fibers"). ${ }^{45}$ Because complex crossing fibers tend to have decreased FA and increased MD, this issue may have influenced our findings. Many approaches have recently been proposed to address this issue, based on high-angular resolution DWI data, ${ }^{46}$ and would help to clarify the nature of WM changes in patients with schizophrenia. Head motion can influence MR imaging studies, in particular when dealing with patients with psychiatric disorders. For this reason, structural images (3D T1 and DTI) were carefully checked, and none showed gross head motion artifacts. Furthermore, the DTI preprocessing used in our study includes a movement correction step (see On-line Appendix for further details). Finally, as in previous studies of patients with schizophrenia ${ }^{47}$ and in accordance with the exploratory nature of the study design, group differences did not survive multiple comparisons correction, suggesting that structural brain changes in schizophrenia are subtle or that some of the significances may have been overestimated. However, we tried to minimize falsepositive findings by using a relatively conservative statistical threshold in the voxel-based morphometry analysis, and by estimating the standard error of the $t$ statistics by using a permutation approach in the TBSS analysis. In TBSS, we permuted the data 5000 times to obtain large, and consequently relatively conservative, standard errors of the $t$ statistics. Such an approach is usually considered robust enough to allow the use of a canonical statistical threshold of $.05 .^{48}$

\section{ACKNOWLEDGMENTS}

The study was partly supported by the Health Authority of the Lombardia Region (Project 153; www.sanita.regione.lombardia.it).

Disclosures: Massimo Filippi—UNRELATED: Board Membership: Teva Pharmaceutical Industries, Genmab A/S; Consultancy: Bayer Schering Pharma, Biogen Idec, Genmab A/S, Merck Serono, Pepgen Corporation, Teva Pharmaceutical Industries; Grants/Grants Pending: Bayer Schering Pharma,* Biogen Idec," Genmab A/S,* Merck Serono, ${ }^{\star}$ Teva Pharmaceutical Industries, ${ }^{\star}$ Fondazione Italiana Sclerosi Multipla, ${ }^{*}$ Italian Ministry of Health, ${ }^{*}$ CurePSP*; Payment for Lectures (including service on speaker bureaus): Bayer Schering Pharma, Biogen-Idec, Genmab A/S, Merck Se- rono, Teva Pharmaceutical Industries; Travel/Accommodations/Meeting Expenses Unrelated to Activities Listed: Bayer Schering Pharma, Biogen Idec, Genmab A/S, Merck Serono, Teva Pharmaceutical Industries. Federica Agosta-UNRELATED: Grants/Grants Pending: Italian Ministry of Health*; Payment for Lectures (including service on speaker bureaus): Bayer Schering Pharma, Biogen Idec, Sanofi Aventis, Serono Symposia International Foundation; Travel/Accommodations/Meeting Expenses Unrelated to Activities Listed: Teva Pharmaceutical Industries. Paolo Valsecchi-UNRELATED: Payment for Lectures (including service on speaker bureaus): Abbott, AstraZeneca, Boehringer-Ingelheim, Bristol-Myers Squibb, Eli Lilly, Innova Pharma, Janssen-Cilag, Lundbeck, Pfizer, Wyeth Lederie. Giancarlo Comi-UNRELATED: Consultancy and Payment for Lectures (including service on speaker bureaus): Novartis, Teva Pharmaceutical Industries, Sanofi-Aventis, Genzyme, MarckSerono, Bayer Schering, Biogen-Dompè, Actelion. Emilio Sacchetti—UNRELATED: Board Membership and Consultancy and Payment for Lectures (including service on speaker bureaus): Abbott, Angelini, AstraZeneca Pharmaceuticals, BoehringerIngelheim, Bristol-Myers Squibb, Danippon Sumitomo Pharma, Eli Lilly, GlaxoSmithKline, Innova Pharma, Italfarmaco, Janssen-Cilag, Lundbeck, Otsuka, Pfizer, Rottapharm, Sanofi-Aventis, Servier, Shire Pharmaceuticals, Stroder, Wyeth Lederle. *Money paid to institution.

\section{REFERENCES}

1. Peters BD, Blaas J, de Haan L. Diffusion tensor imaging in the early phase of schizophrenia: what have we learned? J Psychiatr Res 2010;44:993-1004

2. Levitt JJ, Bobrow L, Lucia D, et al. A selective review of volumetric and morphometric imaging in schizophrenia. Curr Top Behav Neurosci 2010;4:243-81

3. Ellison-Wright I, Bullmore E. Meta-analysis of diffusion tensor imaging studies in schizophrenia. Schizophr Res 2009;108:3-10

4. Cheung V, Cheung C, McAlonan GM, et al. A diffusion tensor imaging study of structural dysconnectivity in never-medicated, firstepisode schizophrenia. Psychol Med 2008;38:877-85

5. Cheung V, Chiu CP, Law CW, et al. Positive symptoms and white matter microstructure in never-medicated first episode schizophrenia. Psychol Med 2011;41:1709-19

6. Gasparotti R, Valsecchi P, Carletti F, et al. Reduced fractional anisotropy of corpus callosum in first-contact, antipsychotic drug-naive patients with schizophrenia. Schizophr Res 2009;108:41-48

7. Guo W, Liu F, Liu Z, et al. Right lateralized white matter abnormalities in first-episode, drug-naive paranoid schizophrenia. Neurosci Lett 2012;531:5-9

8. Pérez-Iglesias R, Tordesillas-Gutiérrez D, Barker GJ, et al. White matter defects in first episode psychosis patients: a voxelwise analysis of diffusion tensor imaging. Neuroimage 2010;49:199-204

9. Zou LQ, Xie JX, Yuan HS, et al. Diffusion tensor imaging study of the anterior limb of internal capsules in neuroleptic-naive schizophrenia. Acad Radiol 2008;15:285-89

10. Szeszko PR, Robinson DG, Ashtari M, et al. Clinical and neuropsychological correlates of white matter abnormalities in recent onset schizophrenia. Neuropsychopharmacology 2008;33:976-84

11. Folstein MF, Folstein SE, McHugh PR. "Mini-mental state". A practical method for grading the cognitive state of patients for the clinician. J Psychiatr Res 1975;12:189-98

12. Wechsler D. WAIS-R Manual. Wechsler Adult Intelligence Scale Revised. New York: The Psychological Corporation; 1981

13. Association AP. Diagnostic and Statistical Manual of Mental Disorders $(D S M-I V)$ 4th ed. Washington, DC: American Psychiatric Association; 1994

14. Sacchetti E, Bocchio-Chiavetto L, Valsecchi P, et al. -G308A tumor necrosis factor alpha functional polymorphism and schizophrenia risk: meta-analysis plus association study. Brain Behav Immun 2007;21:450-57

15. Craig TJ, Bromet EJ, Fennig S, et al. Is there an association between duration of untreated psychosis and 24-month clinical outcome in a first-admission series? Am J Psychiatry 2000;157:60-66

16. Kay SR, Fiszbein A, Opler LA. The positive and negative syndrome scale (PANSS) for schizophrenia. Schizophr Bull 1987;13:261-76

17. Nichols TE, Holmes AP. Nonparametric permutation tests for functional neuroimaging: a primer with examples. Hum Brain Mapp 2002;15:1-25 
18. Innocenti GM, Ansermet F, Parnas J. Schizophrenia, neurodevelopment and corpus callosum. Mol Psychiatry 2003;8:261-74

19. Kyriakopoulos M, Frangou S. Recent diffusion tensor imaging findings in early stages of schizophrenia. Curr Opin Psychiatry 2009;22:168-76

20. Uranova N, Orlovskaya D, Vikhreva O, et al. Electron microscopy of oligodendroglia in severe mental illness. Brain Res Bull 2001;55:597-610

21. Koch K, Wagner G, Dahnke R, et al. Disrupted white matter integrity of corticopontine-cerebellar circuitry in schizophrenia. Eur Arch Psychiatry Clin Neurosci 2010;260:419-26

22. Sun Z, Wang F, Cui L, et al. Abnormal anterior cingulum in patients with schizophrenia: a diffusion tensor imaging study. Neuroreport 2003; 14:1833-36

23. Federspiel A, Begre S, Kiefer C, et al. Alterations of white matter connectivity in first episode schizophrenia. Neurobiol Dis 2006;22:702-09

24. Chance SA, Highley JR, Esiri MM, et al. Fiber content of the fornix in schizophrenia: lack of evidence for a primary limbic encephalopathy. Am J Psychiatry 1999;156:1720-24

25. Guillin O, Abi-Dargham A, Laruelle M. Neurobiology of dopamine in schizophrenia. Int Rev Neurobiol 2007;78:1-39

26. Hubl D, Koenig T, Strik W, et al. Pathways that make voices: white matter changes in auditory hallucinations. Arch Gen Psychiatry 2004;61:658-68

27. Shergill SS, Brammer MJ, Fukuda R, et al. Engagement of brain areas implicated in processing inner speech in people with auditory hallucinations. Br J Psychiatry 2003;182:525-31

28. Seok JH, Park HJ, Chun JW, et al. White matter abnormalities associated with auditory hallucinations in schizophrenia: a combined study of voxel-based analyses of diffusion tensor imaging and structural magnetic resonance imaging. Psychiatry Res 2007;156:93-104

29. Basser PJ, Mattiello J, LeBihan D. MR diffusion tensor spectroscopy and imaging. Biophys J 1994;66:259-67

30. Beaulieu C. The basis of anisotropic water diffusion in the nervous system - a technical review. NMR Biomed 2002;15:435-55

31. Song SK, Sun SW, Ramsbottom MJ, et al. Dysmyelination revealed through MRI as increased radial (but unchanged axial) diffusion of water. Neuroimage 2002;17:1429-36

32. Wheeler-Kingshott CA, Cercignani M. About "axial" and "radial" diffusivities. Magn Reson Med 2009;61:1255-60

33. Bora E, Fornito A, Radua J, et al. Neuroanatomical abnormalities in schizophrenia: a multimodal voxelwise meta-analysis and meta-regression analysis. Schizophr Res 2011;127:46-57
34. Di Rosa E, Crow TJ, Walker MA, et al. Reduced neuron density, enlarged minicolumn spacing and altered ageing effects in fusiform cortex in schizophrenia. Psychiatry Res 2009;166:102-15

35. Vuilleumier P, Armony JL, Driver J, et al. Effects of attention and emotion on face processing in the human brain: an event-related fMRI study. Neuron 2001;30:829-41

36. Thomann PA, Roebel M, Dos Santos V, et al. Cerebellar substructures and neurological soft signs in first-episode schizophrenia. Psychiatry Res 2009;173:83-87

37. Tavano A, Grasso R, Gagliardi C, et al. Disorders of cognitive and affective development in cerebellar malformations. Brain 2007;130:2646-60

38. Blakemore SJ, Wolpert DM, Frith CD. Abnormalities in the awareness of action. Trends Cogn Sci 2002;6:237-42

39. Pantelis C, Velakoulis D, McGorry PD, et al. Neuroanatomical abnormalities before and after onset of psychosis: a cross-sectional and longitudinal MRI comparison. Lancet 2003;361:281-88

40. Peters BD, de Haan L, Vlieger EJ, et al. Recent-onset schizophrenia and adolescent cannabis use: MRI evidence for structural hyperconnectivity? Psychopharmacol Bull 2009;42:75-88

41. Dekker N, Schmitz N, Peters BD, et al. Cannabis use and callosal white matter structure and integrity in recent-onset schizophrenia. Psychiatry Res 2010;181:51-56

42. Bava S, Frank LR, McQueeny T, et al. Altered white matter microstructure in adolescent substance users. Psychiatry Res 2009;173:228-37

43. De Bellis MD, Van Voorhees E, Hooper SR, et al. Diffusion tensor measures of the corpus callosum in adolescents with adolescent onset alcohol use disorders. Alcohol Clin Exp Res 2008;32:395-404

44. Arévalo-Martín A, García-Ovejero D, Rubio-Araiz A, et al. Cannabinoids modulate Olig2 and polysialylated neural cell adhesion molecule expression in the subventricular zone of post-natal rats through cannabinoid receptor 1 and cannabinoid receptor 2. Eur J Neurosci 2007;26:1548-59

45. Alexander DC, Barker GJ, Arridge SR. Detection and modeling of non-Gaussian apparent diffusion coefficient profiles in human brain data. Magn Reson Med 2002;48:331-40

46. Tournier JD, Yeh CH, Calamante F, et al. Resolving crossing fibres using constrained spherical deconvolution: validation using diffusion-weighted imaging phantom data. Neuroimage 2008;42:617-25

47. Miyata J, Sasamoto A, Koelkebeck K, et al. Abnormal asymmetry of white matter integrity in schizophrenia revealed by voxelwise diffusion tensor imaging. Hum Brain Mapp 2012;33:1741-49

48. Good PI. Permutation, Parametric, and Bootstrap Tests of Hypotheses, 3rd ed. New York: Springer; 2005 